\title{
Bioinformation
}

\author{
www.bioinformation.net
}

Hypothesis

\section{Comparative protein modeling of spermidine synthase from Plasmodium falciparum: A potential target for anti-malarial drug therapy}

\author{
Duvvuru Muni Rajasekhara Reddy* \\ Division of biology, University of Fribourg, Fribourg, Switzerland; Email - raja.duvvuru@unifr.ch; \\ * Corresponding author \\ received November 15, 2006; revised December 05, 2006; accepted December 15, 2006; published online December 23, \\ 2006
}

\begin{abstract}
:
Malaria, caused by protozoan parasites of the genus Plasmodium, affects up to 500 million individuals and kills over 1 million people every year. The increasing resistance of the malaria parasites has enforced strategies for finding new drug targets. In recent years, enzymes associated with the polyamine metabolism have attracted attention as drug targets. Cytosolic Plasmodium falciparum spermidine synthase (PfPAPT) is a potential target for antimalarial chemotherapy. Contrasting with the other enzymes involved in the parasite polyamine amine biosynthesis, little information is available about this enzyme, and its crystallographic structure is unknown yet. In this paper I propose a theoretical low-resolution 3D model for PfPAPT based on crystal structure of the Arabidopsis thaliana, by multiple alignment followed by intensive optimization; validation and dynamic simulations in water. Comparison between the active sites of PfPAPT and human PAPT revealed key differences that could be useful for the design of new selective inhibitors of Plasmodium PAPT.
\end{abstract}

Keywords: spermidine synthase; antimalarial drug; drug target; protein modeling

\section{Background:}

Unicellular parasites such as Plasmodium falciparum (malaria), Leishmania major (leishmaniosis), Trypanosoma brucei (sleeping sickness), Trypanosoma cruzi (Chagas disease) and Toxoplasma gonadii (toxoplasmosis) are responsible for the world's most widespread diseases. Malaria remains a major and growing threat to the public health and economic development of countries in the tropical and subtropical regions of the world. [1] Approximately $40 \%$ of the world's population live in areas where malaria is endemic. There are estimated 300-500 million cases and up to 2.7 million deaths from malaria each year, most of them from Africa. In addition, travelers and soldiers are at significant risk for exposure to malaria. Human malaria is caused by infection with intracellular parasites of the genus Plasmodium that is transmitted by Anopheles gambiae mosquitoes. Plasmodium requires two hosts: the Anopheles mosquito for the sexual reproductive stages and humans for the asexual reproductive stages. The infectious sporozoites are injected by the mosquito into blood vessels in the skin. They invade hepatocytes and begin the exoerythrocytic cycle, which generates thousands of merozoites (schizogony). After rupture of the hepatocyte, the merozoites enter the bloodstream and begin the erythrocytic cycle by invading red blood cells, forming trophozoites. Replication in the erythrocyte (schizogony) culminates in rupture and release of a small number of merozoites, which initiate another cycle of replication by infecting other erythrocytes. Among four species of Plasmodium that infect humans, P.falciparum is the most

ISSN 0973-2063

Bioinformation 1(8): 310-313 (2006) lethal. Drug resistance is increasing even to newer antimalarial drugs such as mefloquine. [2] This has led to an urgent need for new anti-malarial drugs both for chemotherapy and prophylaxis.

The current approach in the anti-parasitic drug discovery involves the identification of novel targets from the databases on the parasite genome and metabolic pathways. In recent years, enzymes associated with the polyamine metabolism have attracted attention as drug targets. The ubiquitously distributed polyamines spermidine and spermine are aliphatic polycations that are involved in numerous cellular functions. Changes of intracellular polyamine concentrations were shown to correlate with growth and differentiation processes of pro- and eukaryotes. In polyamine synthesis, spermidine synthase catalyses the transfer of an aminopropyl moiety of decarboxylated S-adenosylmethionine (dcAdoMet) to putrescine, resulting in the formation of spermidine and 5' methylthioadenosine (MTA). [3] During the asexual cycle of Plasmodium falciparum within the host erythrocyte, the parasite induces a stage-dependent elevation in the levels of polyamines by increased metabolism and uptake of extracellular pools. The enzymes associated with biosynthetic pathway of polyamines exhibit features that differ significantly between the parasites and the human host. Therefore it is conceivable that exploitation of such differences can lead to the design of new inhibitors that will selectively kill the parasites while exerting minimal or at 


\section{Bioinformation}

\section{www.bioinformation.net}

Hypothesis

least tolerable, effects on the parasite-infected patient. [4] The gene encoding spermidine synthase was cloned recently from P.falciparum. Northern and Western blot analyses revealed a stage specific expression during the erythrocytic schizogony with the maximal amount of transcript and protein in mature trophozoites. The spermidine synthase polypeptide of 321 amino acids has a molecular mass of $36.6 \mathrm{kDa}$ and contains an $\mathrm{N}$-terminal extension of unknown function that, similarly, is also found in certain plants but not in animal or bacterial orthologues. In transient expression analysis experiments the enzyme catalyses the transfer of an aminopropyl group from decarboxylated S-adenosylmethionine [dcAdoMet] onto putrescine. In contrast to mammalian spermidine synthases, spermidine can replace to some extent putrescine as the aminopropyl acceptor. Hence, P. falciparum spermidine synthase has the capacity to catalyse the formation of spermine. [5] The functional differences observed could be due to the structural differences and might be further exploited for the development of the antimalarial drugs. Therefore it is our interest to study the structural features of spermidine synthase from P.falciparum. The structure of spermidine synthase from P.falciparum is unknown. In the absence of the experimentally determined structure, molecular modeling is usually the method of choice, when there is clear relationship of homology between the sequence of target protein and at least one known structure.

\section{Methodology:}

The single letter amino acid sequence of P.falciparum PAPT (Q8II73) was retrieved from SWISS PROT [6] and taken as target sequence. The modeling of 3D structure of
PfPAPT followed a stepwise procedure, starting with a template structure search from PDB (protein databank) [7], related to the target sequence using BLASTP. [8] From a number of hits, a potential template structure (PDB-ID: IXJ5), representing the structure of spermidine synthase form Arabidopsis thaliana was taken as template for model building. The structure of the multi-substrate adduct inhibitor AdoDATO was taken from the crystal structure of Thermotoga Maritima (PDB-ID: 1JQ3).

\section{Model Building, evaluation and active site analysis}

The template and target sequences were aligned using the MALIGN script. After careful examination for the potential alignment errors, the automated comparative protein modelling program MODELLER7v7 was used to build the model. [9] In the first step of model building, distance and dihedral angle restraints on the target sequence were derived from its alignment with the template 3D-structure. The spatial restraints and the energy minimization steps were performed with the CHARMM22 force field for proper stereochemistry of proteins. Then, optimization of the model was carried out by the molecular dynamics simulated annealing method. The modeled structure with minimum objective function was taken and evaluated by the PROCHECK. [10] The overall stereochemical quality of the protein was assessed by Ramachandran plot analysis. [11] The modeled 3D structure of PfPAPT was used for docking with PAPT inhibitor to find the active pocket of ligand binding using the Hex4.2 [12] macromolecular docking program with default parameters of the docking module.
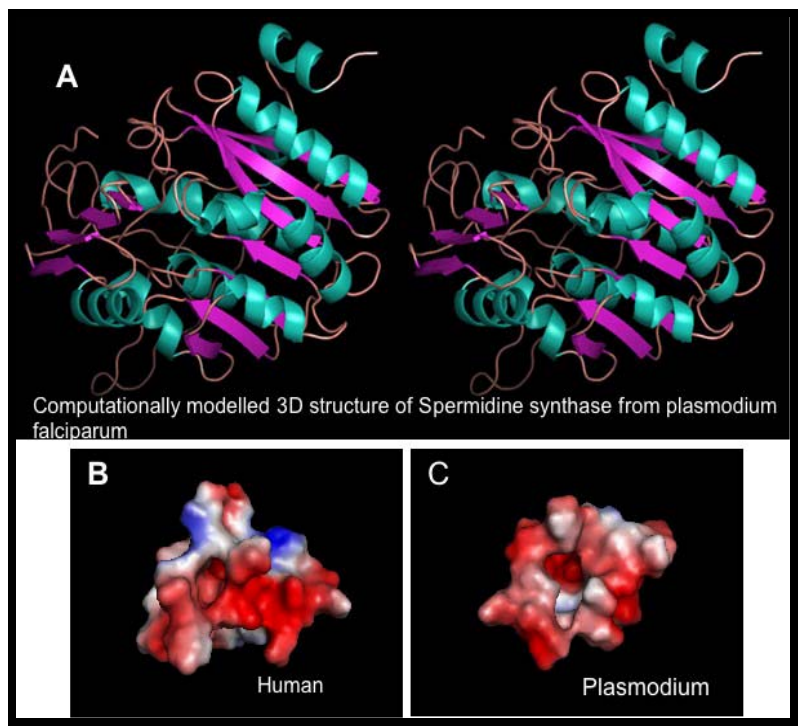

Figure 1: (A) Predicted model of Plasmodium falciparum spermidine synthase [PDB ID: 2ce5] in sterio view. (B \& C) Electrostatic potential differences mapped on to the active site resides with-in the $5 \mathrm{~A}^{\mathrm{o}}$ distance

ISSN 0973-2063

Bioinformation 1(8): 310-313 (2006)
311

Bioinformation, an open access forum (c) 2006 Biomedical Informatics Publishing Group 


\section{Bioinformation}

www.bioinformation.net

\begin{abstract}
Results and discussion:
The result of the protein-protein BLAST search for a suitable template structure related to the target sequence (PfPAPT) showed Arabidopsis thaliana spermidine synthase with highest sequence similarity (47\%), as the most suitable template for modeling. In total twenty five models were generated by MODELLER 7V7, and the model with the lowest objective function (967.8491) was selected as the best and subjected to "internal" evaluation of self-consistency checks such as stereochemical check to find the deviations from normal bond lengths, dihedrals and non-bonded atom-atom distances. The PROCHECK Ramachandran plot analysis shows $91.3 \%$ of the residues are in the core region, $6.4 \%$ in the allowed and $2.3 \%$ in the generously allowed regions. No residues were found in the disallowed region of the Ramachandran plot showing that no residues have a disallowed conformation. The Goodness factors (G-factors), from the PROCHECK results summary show the quality of covalent and overall bond/angle distances. The observed G-factors for the present model were -0.10 for dihedrals, -0.17 for covalent and overall 0.01 . The comparable Ramachandran plot characteristic and the goodness factors confirm the quality of the modelled structure. The evaluated final model has been deposited in PDB and is now publicly accessible [PDB ID: 2ce5]. The protein structure predicted is displayed in figure 1A. The overall topology of the modelled spermidine synthase from Plasmodium falciparum, like other members of the enzyme family, consists the typical two domains. The larger C-terminal catalytic core domain consists of seven-stranded $\beta$-sheets flanked by nine $\alpha$-helices. A PAPT family signature V-[LA]-[LIV] [2]-G-G-G-X[2][LIV]-X-E (where $X$ is any amino acid) which is previously defined in [PROSITE entry PS01330] is identified in the core catalytic domain at C-terminus between $\beta 8-\alpha 6$. The RMSD (Root Mean Square Deviation) between predicted model and template is 0.4 angstrom.
\end{abstract}

The structure of T.maritima and human PAPT were determined in complex with inhibitor AdoDATO and with AdoMet, respectively. The structures demonstrate a high level of conservation of the overall topology. Thus the structures of T.maritima and human PAPT could be superimposed on the P.falciparum PAPT with rmsd for the $\mathrm{C}-\alpha$ atoms of around $1 \mathrm{~A}^{0}$. The largest differences between the P.falciparum and T.maritima were found in the regions of helix $\alpha 3, \alpha 4$, and $\alpha 6$ (residues 142-151, 168-177 and 230-242, respectively. A superimposition of the structures of human and T.maritima PAPT shows that the differences are maintained from the $\alpha 3$ and $\alpha 6$, while $\alpha 4$ superimposes well on the corresponding helix from the T.maritima enzyme. Taking into account that $\alpha 4$ builds up part of the substrate binding cleft, the differences between human and

ISSN 0973-2063

Bioinformation 1(8): 310-313 (2006)
P.falciparum enzymes may be of interest for future design of plasmodium specific inhibitors.

Search for the ligand binding pocket using multisubstrate adduct inhibitor AdoDATO, resulted in the identification of active site. Active site residues were then searched for the differences and the electrostatic potentials were mapped on to the surface. Amino acid residues with in the $5 \mathrm{~A}^{0}$ distance surrounding the Active site display qualitative electrostatic potential differences (Fig1 B and C). The structure of T.maritima PAPT in complex with AdoDATO shows that the putricine-binding site is a hydrophobic cavity, which includes two negatively charged sites responsible for anchoring the amino acid groups of the molecule. In particular, Asp170 (T.maritima numbering) has been proposed to be responsible for deprotonating the attacking amino group of putricine, where as Tyr76 and Ser171 are thought to be involved in binding and proper orientation of the diamine. The corresponding residues in the P.falciparum are Tyr90, Asp184 and Ser185. In total 20 amino acid residues are involved in interaction with the inhibitor of these 16 are conserved, where as four have been exchanged in P.falciparum. These differences observed in the active site architecture could be used to design specific inhibitors.

\section{Utility:}

Despite the progress made in anti-malarial research, control of malaria has been severely compromised by the development of malarial parasites resistant to nearly all anti-malarial drugs used for prophylaxis and treatment. In order to provide a new target for the development of antimalarial drug discovery I have chosen plasmodium polyamine biosynthetic pathway enzyme spermidine synthase. A molecular model of the spermidine synthase from Plasmodium falciparum is documented in this study. The model is believed to provide valuable insights towards the design of an inhibitor specific to the spermidine synthase of Plasmodium falciparum for the treatment of malaria, using the provided active site location and conformations.

\section{References:}

[01] K. K. Manhani, et al., J Mol Model, 12:42 (2005) [PMID: 16096806]

[02] W. Milhous, Bull Soc Pathol Exot., 94:149 (2001) [PMID: 16579068]

[03] T. Thomas \& T. J. Thomas, Cell Mol Life Sci., 58:244 (2001) [PMID: 11289306]

[04] O. Heby, et al., Biochem Soc Trans., 31:415 (2003) [PMID: 12653650]

[05] N. Haider, et al., Mol Biochem Parasitol., 142:224 (2005) [PMID: 15913804]

[06] http://www.expasy.org/

[07] http://www.rcsb.org/pdb/ 


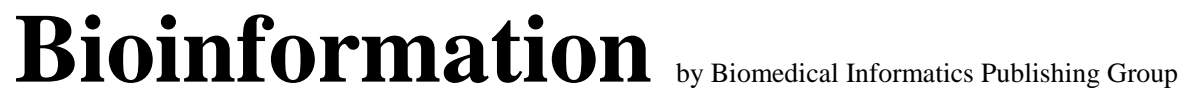

open access

\section{www.bioinformation.net}

\section{Hypothesis}

[08] http://www.ncbi.nlm.nih.gov/blast/

[09] A. Sali \& T. L. Blundell, J. Mol. Biol., 234:779 (1993) [PMID: 8254673]

[10] R. A. Laskowski, et al., J Appl Crystallogr., 26:283 (1993)
[11] G. N. Ramachandran, et al., J. Mol. Biol., 7:95 (1963) [PMID: 13990617]

[12] D. W. Ritchie \& G. J. L. Kemp, Proteins, 39:178 (2000) [PMID: 10737939]

Edited by P. Kangueane

Citation: Reddy, Bioinformation 1(8): 310-313 (2006)

License statement: This is an open-access article, which permits unrestricted use, distribution, and reproduction in any medium, for non-commercial purposes, provided the original author and source are credited. 